\title{
Trockengewicht und Kaloriengehalt sich entwickelnder Heringseier
}

\author{
G.-A. Paffenhöfer und H. Rosenthal \\ Biologische Anstalt Helgoland, Meeresstation, Helgoland, und Zentrale, Hamburg
}

\begin{abstract}
Dry weight and caloric content of developing herring eggs. Dry weight and caloric content of eggs from spring spawning western Baltic Sea herrings have been determined employing the bomb calorimetry technique. Water temperature throughout incubation was kept at $8.0^{\circ} \pm 0.2^{\circ} \mathrm{C}$. Average dry weight and caloric content values of one pist fertilized egg are $0.170 \mathrm{mg}$ ( $0.124 \mathrm{mg}$ without chorion) and $0.748 \mathrm{cal}$ (without chorion), respectively. The caloric content per egg decreases progressively during the incubation period (Fig. 2). The same holds for the dry substance of the egg (Fig. 1). Energy extraction efficiency related to dry weight is $67.8 \%$, the efficiency related to dry organic substance $70.4 \%$, and the caloric efficiency $63.7 \%$.
\end{abstract}

\section{EINLEITUNG}

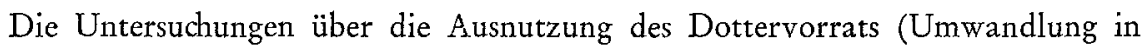
Embryosubstanz und biologisch nutzbare Energie) während der Inkubationszeit und Larvalphase bei Fischen beruhen bisher fast ausschließlich auf gewichtsbezogenen Analysen (GRAY 1926, 1928a, b; Blaxter \& Hempel 1966). Für die Heringsbrut der Kieler Frühjahrslaicher fanden Blaxter \& HeMPEL (1966) eine Nutzungsrate von $74 \%$, für die etwas größeren Buchan-Eier $63 \%$ und für die Eier der Downs-Heringe $52 \%$.

Die vorliegende Arbeit befaßt sich mit dem energetischen Stoffumsatz bei Eiern und Larven des Herings (Clupea harengus L.) aus der westlichen Ostsee (Frühjahrslaicher). An diesem Objekt erschien die Ermittlung des Energiegehalts und Energieverbrauchs besonders wünschenswert, weil zahlreiche vergleichbare Daten über die Ausnutzung des Dottervorrats auf gewichtsanalytischer Basis zur Verfügung stehen (BLAXTER \& HEMPEL 1966).

\section{MATERIAL UND METHODEN}

\section{Material}

Die Erbrütungsversuche wurden in mehreren Ansätzen an küstenlaichenden Frühjahrsheringen der Ostsee (Schleimündung) durchgeführt. Die benutzten Eiproben stammten aus den Versuchsreihen vom 23. März (A) und 1. April 1967 (B). Die Totallängen der Weibchen betrugen: $28 \mathrm{~cm}$ (23. März); 28, 26, 27 und $27 \mathrm{~cm}$ (1. April 1967). Das Sperma wurde von 4 bis 8 Männchen entnommen. Die Befruchtungsversuche fanden unmittelbar nach der Anlandung (etwa 1 Stunde nach dem Fang) statt. 


\section{Methoden}

Die Befruchtung erfolgte in einer Plastikschale in verdünntem Meerwasser mit einem Salzgehalt von 15\%. Die Wassertemperatur betrug 8,0 $\pm 0,5^{\circ} \mathrm{C} \mathrm{(23.} \mathrm{März} \mathrm{und}$ 1. April). Die Eier wurden abgestreift und klebten unter Wasser auf den eingelegten Mattglasscheiben fest. Etwa 30 Minuten nach dem Abstreifen wurde das in der Schale überstehende Wasser dekantiert und das in 15\% S aufgelöste Sperma hinzugegeben. Die Eier verblieben in dieser Lösung etwa 1 bis $1 \frac{1 / 2}{2}$ Stunden. Danach wurden die Mattglasscheiben in frischem Meerwasser von 15\% gründlich abgespült und in die Transportgefäße überführt. Der Transport von Schleswig nach Hamburg dauerte etwa 3 Stunden. Während des Transportes trat eine geringfügige Temperaturerhöhung $(0,5$ bis $0,8^{\circ} \mathrm{C}$ ) ein. Unmittelbar nach dem Transport wurden die Eier in Hälterungsgefäße überführt, die in einem Temperierbad während der Dauer der Erbrïtungsversuche auf $8,0 \pm 0,2^{\circ} \mathrm{C}$ konstant gehalten wurden. Das Kulturwasser wurde täglich erneuert. Um den Aufwuchs von Mikroorganismen auf der Eioberfläche zu mindern, wurden die Eier in Abständen von 1 bis 3 Tagen mit einem weichen Pinsel vorsichtig gesäubert. Im Aquarium betrug die Lichtintensität am Mittag je nach Witterung 50 (bedeckt) bis 150 Lux (sonnig).

Das Temperierbad, in dem sich die Kulturschalen befanden, wurde mit einer Platte abgedeckt, um den Lichteinfall weiter zu reduzieren.

Die zur Bestimmung des Energiegehaltes (cal) benutzten Eier wurden mittels einer Rasierklinge von den Mattglasscheiben abgelöst und gezählt. In der Regel enthielt eine Probe 100 bis 200 Eier oder 60 bis 200 Larven. Die entnommene Probe wurde etwa 40 Sekunden in destilliertem Wasser belassen, um das anhaftende Salzwasser zu entfernen, anschließend 12 bis 15 Stunden bei $60^{\circ} \mathrm{C}$ und 1 Stunde bei $80^{\circ} \mathrm{C}$ getrocknet. Die getrockneten Proben wurden in Exsikkatoren (Silicagel) aufbewahrt, und spätestens 14 Tage nach der Trocknung in einem PARR 1412 Semimicrocalorimeter verbrannt. Die Proben wogen zwischen 5 und $25 \mathrm{mg}$ (Trockengewicht). Bei der Verbrennung dieser Gewichtsmengen ist nur eine geringe Temperatursteigerung zu erwarten, was zu ungenauen Meßergebnissen führt. Um einen hinreichend hohen Temperaturanstieg zu erhalten, wurden jeder Probe etwa $125 \mathrm{mg}$ Benzoësäure hinzugefügt. Das Gewicht der getrockneten Proben und der Benzoësäure wurde auf einer SARToriusAnalysenschnellwaage 2604 (Genauigkeit $\pm 0,01 \mathrm{mg}$ ) bestimmt. Die Proben wurden jeweils zweimal gewogen. Des weiteren wurde der Salz- und Aschegehalt verschieden alter Eier und Larven ermittelt.

\section{ERGEBNISSE}

\section{Trockengewicht}

Generell zeigt das Eitrockengewicht, in Ubereinstimmung mit den Befunden von BlaXter \& HEMPEL (1963), eine lineare Gewichtsabnahme während der Inkubationszeit (Abb. 1, Regression b $=-2,385$, Versuchsreihen A und B).

Der Ausgangswert liegt jedoch mit 0,124 mg pro Ei (ohne Chorion) um etwa $30 \%$ 
höher. Nach Hempel (1962) kann bei den Kieler Heringen das Eitrockengewicht zwischen 9 und $20 \mathrm{mg} / 100$ Eier (mit Chorion) schwanken. Das in Versuchsreihe B gefundene Trockengewicht von $17,0 \mathrm{mg} / 100$ Eier liegt damit unterhalb der Maximalgrenze.

Tabelle 1

Abnahme des Trockengewichts und der organischen Substanz in Ei (ohne Chorion) und frisch geschlüpfter Larve von Clupea harengus. $\mathrm{A}=$ Versuchsreihe vom 23. März; $\mathrm{B}=$ Versuchsreihe vom 1. April 1967. Durchschnittswerte von jeweils 2 bis 5 Proben

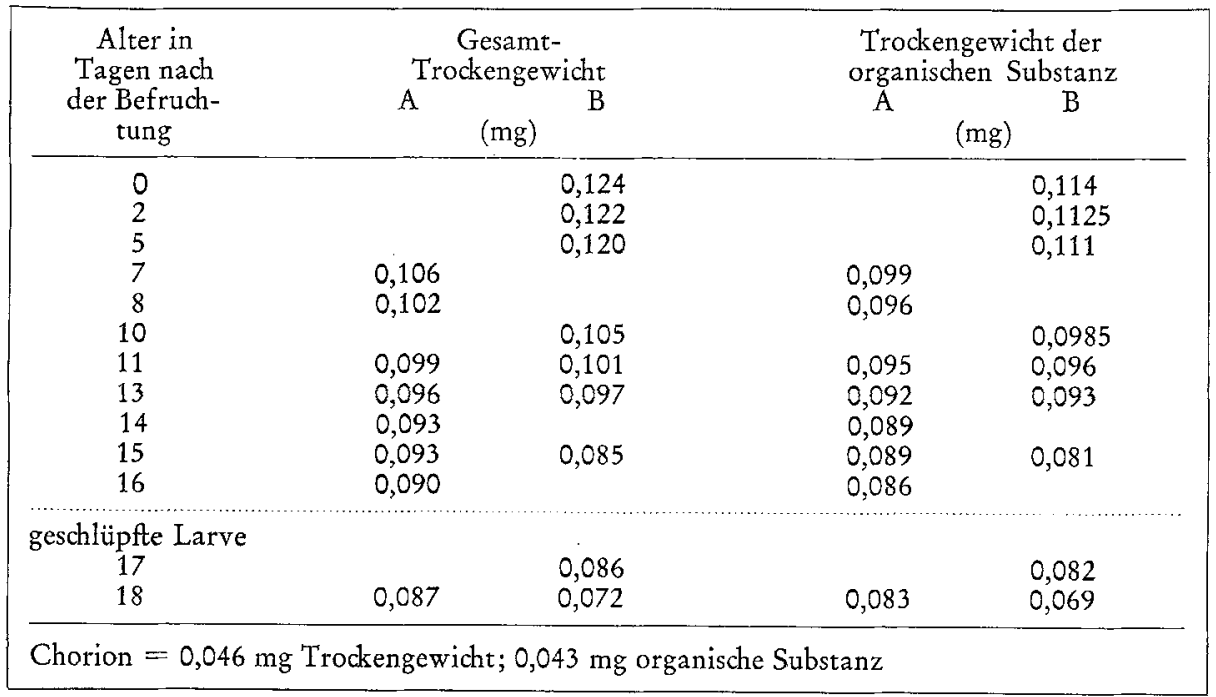

Tabelle 2

Aschegehalt verschieden alter Eier und frischgeschlüpfter Larven von Clupea harengus (in \% vom Eitrockengewicht)

\begin{tabular}{|cc|}
\hline $\begin{array}{c}\text { Alter in Tagen nach } \\
\text { der Befruchtung }\end{array}$ & \% Aschegehalt \\
\hline 0 & 7,4 \\
5 & 6,6 \\
11 & 5,4 \\
\hline geschlüpte Larve & 4,6 \\
\hline 16 & 6,3 \\
\hline Chorion & 6 \\
\hline
\end{tabular}

Betrachtet man in Abbildung 1 die Mittelwerte der einzelnen Beobachtungsintervalle für sich, so scheint eine geringfügige Beschleunigung in der Trockengewichtsabnahme (besonders gegen Ende) der Inkubationszeit einzutreten (punktierte Linie). Anhand der geringen Zahl der Beobachtungswerte ist jedoch nicht nachzuweisen, ob eine derartige Tendenz tatsächlich vorliegt.

In Tabelle 1 wird für die Versuchsreihen A und B das Eitrockengewicht und das 


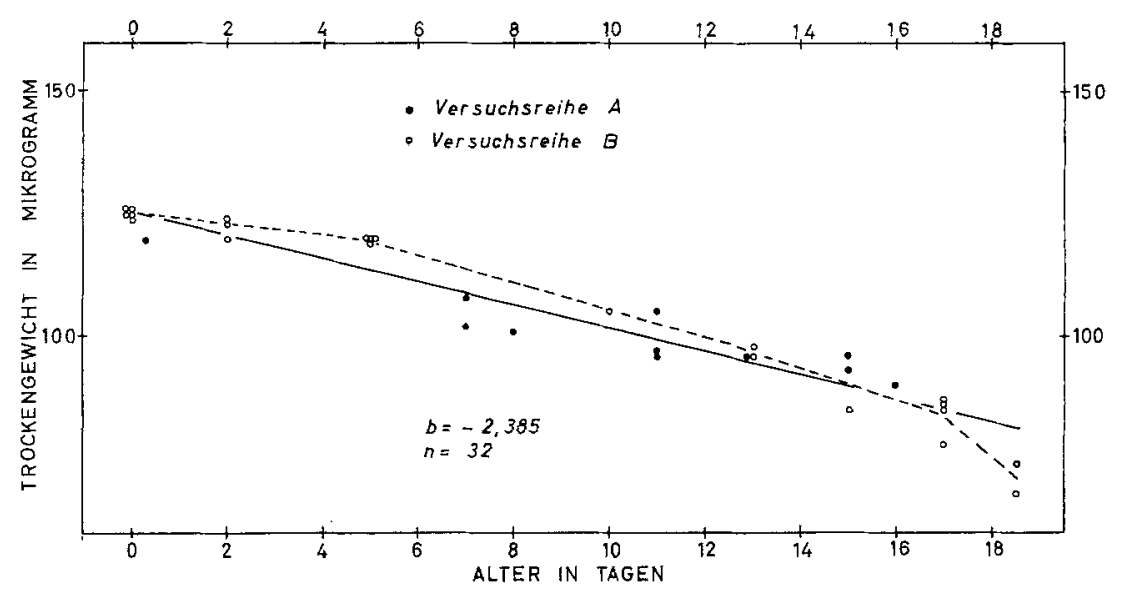

Abb. 1: Gewichtsabnahme von Ei und Larve des Herings (Clupea havengus) während der Entwicklung bei $8^{0} \pm 0,2^{\circ} \mathrm{C}$. Proben aus 2 Versuchsreihen mit jeweils 100 bis 200 Eiern beziehungsweise 60 bis 200 Larven

Trockengewicht der frischgeschlüptten Larven sowie das jeweils zugehörige Gewicht der organischen Substanz pro Ei und Larve getrennt aufgeführt. Da der Aschegehalt sich während der Entwicklung ändert, ergibt sich eine geringfügige prozentuale Änderung der organischen Substanz bezogen auf das Eitrockengewicht: die Abnahme der organischen Substanz von der Befruchtung bis zum Schliipfen ist mit $24,6 \%$ geringer als die Verminderung des Eitrockengewichts $(27,4 \%$ ).

Um das Gewicht und den Energiegehalt des Chorions zu bestimmen, wurden für jede Probe jeweils 200 bis 300 Eier aufgeschnitten, die Embryonen und die perivitelline Flüssigkeit entfernt und anschließend zwei Minuten lang in destilliertem Wasser ausgespült. Der Anteil des Chorions (0,046 mg/Ei) beträgt im Mittel $27 \%$ des Eitrockengewichts $(0,170 \mathrm{mg} / \mathrm{Ei})$. Dieser Wert entspricht ungefähr den Angaben von Blaxter \& HEMPEL (1966), die für die Eier der Kieler Heringe einen Choriongewichtsanteil von 31 bis $33 \%$ ermittelten.

\section{Energiegehalt}

Unmittelbar nach der Befruchtung beträgt der Kaloriengehalt eines einzelnen Heringseies ohne Chorion im Mittel 0,748 cal (Versuchsreihe B). Frischgeschlüpfte Larven (16 Tage nach der Befruchtung) haben einen Kaloriengehalt von etwa 0,500 cal. Zwei Tage nach dem Schlüpfen besitzen die Larven nur noch 0,404 cal pro Individuum. Bis zum Schlüpfen werden zur Deckung des Gesamtstoff wechsels eines Heringeies etwa ein Drittel der ursprünglichen Dotterenergiemenge $(0,248 \mathrm{cal})$ verbraucht. Bezieht man den Energieverbrauch auf Kalorien pro Gramm organischer Substanz, so vermindert sich der Kaloriengehalt um $645 \mathrm{cal}$ (von 6585 auf 5940).

Der Kaloriengehalt pro Ei (ohne Chorion) nimmt im Laufe der Embryonalentwicklung nicht linear, sondern mit leicht ansteigender Geschwindigkeit ab (Abb. 2). 


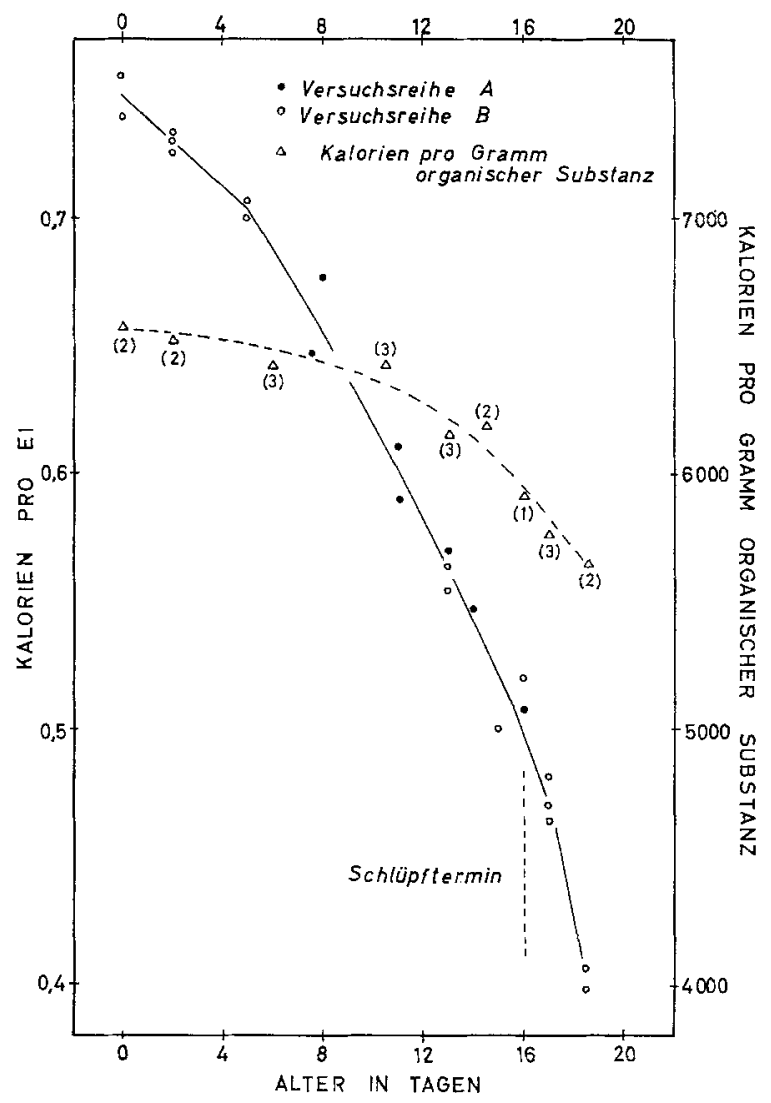

Abb. 2: Energieabnahme im Ei während der Inkubationszeit von Clupea barengus. Erbrütungstemperatur $8,0^{\circ} \pm 0,2^{\circ} \mathrm{C}$. Kaloriengehalt pro $\mathrm{Ei}$ (ohne Chorion) und Kaloriengehalt pro Gramm organischer Substanz. (Zahlen in Klammern = Anzahl der Proben)

Diese Tendenz wird deutlicher, wenn der Energiegehalt in Kalorien pro Gramm organischer Substanz ausgedrüdkt wird (ohne Chorion).

Das Chorion eines einzelnen Eies enthält im Mittel 0,180 cal. Der Kaloriengehalt des Chorions bezogen auf die organische Substanz in Gramm wurde mit $4083 \mathrm{cal}$ ermittelt.

Die Einzelbestimmungen des Chorions unterschiedlich alter Eier weisen eine nur geringe Streuung auf (4089 cal, $4095 \mathrm{cal}$ : 4 Tage alt; $4066 \mathrm{cal}$ : 13 Tage alt).

\section{DISKUSSION}

Das Eitrockengewicht nimmt während der Inkubationszeit linear ab (Abb. 1). Demgegenüber steigt der Energieverbrauch zunächst exponentiell mit zunehmendem Alter an (Abb. 2). Einen ähnlichen Kurvenverlauf fand Lasker (1965) für Sardinops caerulea (Abb. 3), der für das unbefruchtete Ei einen Kalorienwert des Eidotters von 
$5807 \mathrm{cal} / \mathrm{g}$ organischer Substanz ermittelte. Demgegenüber beträgt der Kaloriengehalt der befruchteten Heringseier $6585 \mathrm{cal} / \mathrm{g}$ organischer Substanz. Die Stoffwechselrate erreicht bei Sardinops caerulea jedoch vor dem Ende der Inkubation ihren höchsten Wert, während sie beim Hering nach dem Schlüpfen weiter ansteigt. Allerdings ist ein

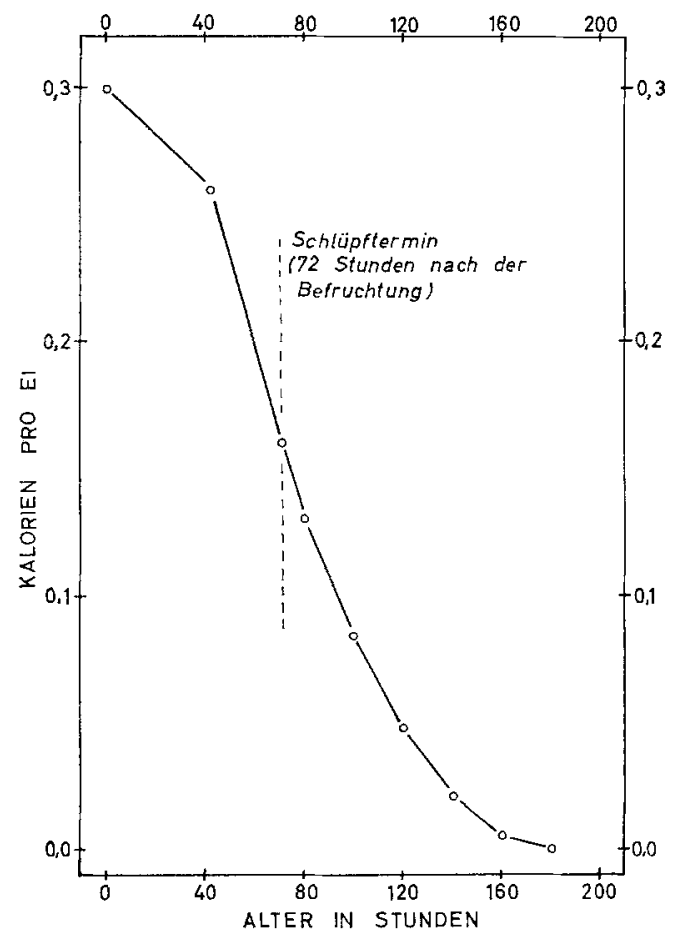

Abb. 3: Energieabnahme im Ei während der Entwicklung von Sardinops caerulea bei $14^{\circ} \mathrm{C}$. (Nach Daten von LASKER 1962)

Vergleich auf Grund der vorliegenden Informationen kaum möglich, zumal beide Arten bei verschiedenen Temperaturen erbrütet wurden.

Die Ausnutzung der Dotterenergie während der Inkubationszeit wird nach BLAXTER \& Hempel (1966) durch die Relation

$$
\frac{\text { gebildeter Organismus (Larve ohne Dotter) }}{\text { gebildeter Organismus + verbrauchte Dottersubstanz }}
$$

wiedergegeben. Bei einem Larventrockengewicht von 0,087 mg (Larve mit Dotter), $0,010 \mathrm{mg}$ (Dotter), 0,077 mg (Larve ohne Dotter), beträgt die auf das Trockengewicht bezogene Ausnutzung

$$
\frac{0,077}{0,077+0,037}=67,8 \%
$$

Die Ausnutzung bezogen auf die organische Substanz errechnet sich mit

$$
\frac{0,074}{0,074+0,031}=70,4 \%
$$


Der kalorische Wert (Kaloriengehalt der Larve am 16. Tag der Inkubation) wurde mit 0,498 cal ermittelt. Kalkuliert man den Kaloriengehalt des Dotterrestes nach den Daten von Blaxter \& Hempel (1963) für den 16. Inkubationstag, so läßt sich der Kaloriengehalt des gebildeten Organismus auf 0,439 cal berechnen. Die kalorische Ausnutzung beträgt demnach

$$
\frac{0,439}{0,439+0,250}=63,7 \%
$$

Neben den Gewichtsverlusten tritt gleichzeitig eine Verringerung des absoluten kalorischen Gehaltes (Kalorien pro Gramm organischer Substanz) während der Entwicklung ein. Daher ist die kalorische Ausnutzung deutlich niedriger als die trockengewichtsbezogene. Energieverbrauch und Nutzungsrate können temperaturabhängig sein. In weiteren Versuchen soll geklärt werden, auf welchem Stadium der Embryonalentwicklung der größte Energieverbrauch zu erwarten und bei welcher Temperatur die Nutzungsrate optimal ist.

\section{ZUSAMMENFASSUNG}

1. Trockengewicht und Kaloriengehalt sich entwickelnder Eier von küstenlaichenden Heringen der westlichen Ostsee wurden bestimmt.

2. Das durchschnittliche Trockengewicht eines einzelnen Eies beträgt kurz nach der Befruchtung $0,170 \mathrm{mg}$ (ohne Chorion 0,124 mg).

3. Der durchschnittliche Kaloriengehalt eines einzelnen Eies ohne Chorion liegt direkt nach der Befruchtung bei 0,748 cal. Bis zum Zeitpunkt des Schlüpfens sinkt der Energiegehalt auf $0,500 \mathrm{cal} \mathrm{ab}$.

4. Der Energiegehalt des Dotters frisch befruchteter Eier beträgt im Mittel $6585 \mathrm{cal}$ pro Gramm organischer Substanz.

5. Die trockengewichtsbezogene Ausnutzung beträgt 67,8\%, die Ausnutzung bezogen auf die organischer Substanz 70,7\%, die kalorische Ausnutzung 63,7\%.

Die Arbeit wurde mit finanzieller Unterstïtzung der Deutschen Forschungsgemeinschaft durchgeführt. Fräulein C. Boedecker und Herrn J. Marschall danken wir für die Hilfe bei der Durchführung der Versuche und Herstellung der Abbildungen. Herrn Professor Dr. O. KINNE danken wir für die kritische Durchsicht des Manuskripts.

\section{ZITIERTE LITERATUR}

Blaxter, J. H. S. \& Hempel, G., 1963. The influence of egg size on herring larvae (Clupea barengus). J. Cons, perm. int. Explor. Mer 28, 211-240.

- - 1966. Utilization of yolk by herring larvae. J. mar. biol. Ass. U.K. 46, 219-234.

Gray, J., 1926. The growth of fish. 1. The relationship between embryo and yolk in Salmo fario. J. exp. Biol. 4, 215-225.

- 1928a. The growth of fish. 2. The growth-rate of the embryo of Salmo fario. J. exp. Biol. $6,110-124$.

- 1928b. The growth of fish. 3. The effect of temperature on the development of the eggs of Salmo fario. J. exp. Biol. 6, 125-130. 
Hempel, G., 1962. Über die biologische Bedeutung der Eigröße beim Hering (Clupea barengus L.). Hamburg, Habii.-Schr., 99 pp.

LASKER, R., 1962. Efficiency and rate of yolk utilization by developing embryos and larvae of the Pacific sardine Sardinops caerula (GIrard). J. Fish. Res. Bd Can. 19, 867-875.

- 1965. The physiology of Pacific sardine embryos and larvae. Rep. Calif. coop. oceanic Fish. Invest. 10, 96-101. 